\title{
MORPHOLOGY OF KOCH FRACTAL ANTENNA
}

\author{
Vinita Mathur ${ }^{1}$, Dr. Manisha Gupta ${ }^{2}$ \\ ${ }^{1}$ Research Scholar, Dept. of ECE, JECRC University, Jaipur \\ vinitamathur12@gmail.com \\ ${ }^{2}$ Associate Professor, Dept. of Physics, JECRC University, Jaipur \\ manisha.gupta@jecrcu.edu.in
}

\begin{abstract}
Antenna is paramount element for the radio communication entity using radio frequency and microwaves. In twenty-first century wireless communication systems, there is a demand for wider bandwidth, multiband and low profile antennas for both commercial and military purposes. This has initiated antenna analysis in various directions; one of them is using fractal shaped antenna elements. Fractal concepts have emerged and advanced as a unique technique for designing compact UWB antennas, because of the self-similarity and space-filling attributes. In a fractal antenna, the multiple frequencies of operation depend on the total dimensions of the design and the scale factor. There are varied fractal geometries that have been found to be favorable in developing novel and new models for antennas. In this paper we will be discussing one of the types of fractal antenna i.e. Koch structure. Koch antenna can be designed with a triangle, rectangle and pentagon as its initiator. There geometry and parameters are been analyzed in this paper.
\end{abstract}

\section{Indexing terms/Keywords}

Antennas, Wireless Communication, Fractal, Ultra-Wideband, Koch.

\section{Council for Innovative Research}

Peer Review Research Publishing System

Journal: INTERNATIONAL JOURNAL OF COMPUTERS \& TECHNOLOGY

Vol 13, No. 2

editor@cirworld.com

www.cirworld.com, www. ijctonline.com 


\section{INTRODUCTION}

In wireless communication antennas are the backbone and almost everything, the world could have not reached at this age of technology without them. Some of them are Parabolic Reflectors, Patch Antennas, Slot Antennas, and Folded Dipole Antennas. Each variety of aerial is good in its own means and applications. Recent telecommunication systems desire antennas with large bandwidths and minor size than traditionally possible.

Patch antennas play a very valid role in today' world of wireless connection systems. A conventional Microstrip fabrication technique [1] is been used in the construction of a simple patch antenna. Multilayer stacked patches or air filled substrate has been used for successful implementation of different broad band techniques [2]. Bulky size and frequency of operation were some of its limitations. In the next stage of development came flat profile printed antennas-the microstrip antenna, the stripline slot antenna, the cavity backed printed antenna and the printed dipole antenna [3]. Wider bandwidth, multiband and low profile antennas are in great demand in modern wireless communication systems and increasing of other wireless applications for both commercial and military applications. This has initiated antenna research in various directions; one of them is using fractal shaped antenna elements.

The term fractal was originally perceived by Mandelbrot [4] to describe a family of sophisticated contour that have repeatability in their architectures. The main encouragement of fractal modeling comes from the many fractal figures present in nature. For example, fractals have been excellently used to model composite natural entities such as clouds, boundaries, coastlines, galaxies, mountain ranges, snowflakes, ferns, trees, leaves and many more geometrical objects as shown in figure 1.For military and commercial utilization the antennas should have the features of multiband behavior, compact size, conformal and low profile. Fractal geometries with their complicated repeated nature demonstrate these required qualities. For multiband applications there suitability of fractal antenna is thoroughly discussed in $[5,6]$.
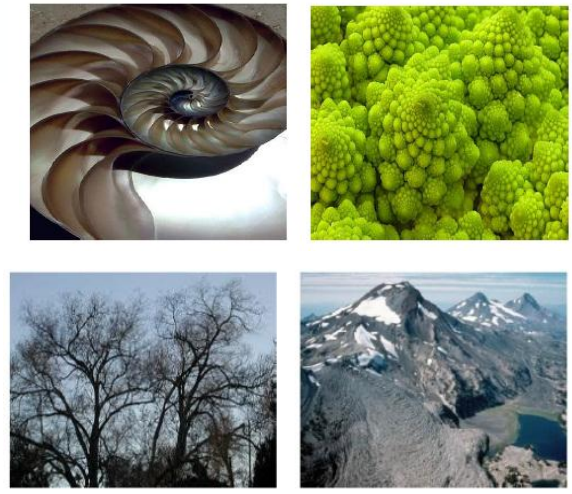

Figure 1 Examples of fractals that can be found in nature

A fractal is a rough or divided measurable contour that can be split into parts, each of which is a diminished-size copy of the whole. Loops, dipoles, monopoles, patch, slot antennas, and antenna arrays, fractal techniques have been applied to all these. EM waves can be picked up by any arbitrarily random shape. These fractal dimensions of the geometry can be linked with antenna features. Slots have to be created for the general antennas for the frequency for which they are to be used-and thus they only work well at that particular frequency. This causes the fractal antenna an excellent design for wideband[7].

Euclidean geometry holds the foundation to the analysis and design of antenna systems in historic approaches. Fractals geometries fall between Euclidean geometries which are limited to points, lines, sheets, and volumes. Therefore, a line that approaches a sheet can be a fractal. Curves that are electrically very long are led by these space-filling properties,but they fit into a close physical area. Antenna elements are miniaturized by this property.Advancement of new types of antennas that employ fractal rather than Euclidean geometric concepts has been a recent concern in the possibility of designing new varieties of antennas. Fractal geometry is development of academic geometry; its novel launch contributes engineers with the fantastic moment to analyze a practically vast number of formerly nonexistent structures for probable use of the evolution of distinct and new antenna formation [8].

In many branches of science and engineering, a wide variety of applications for fractals continue to be found, fractal electrodynamics is one of them. In this fractal geometry is united with electromagnetic theory for the idea of evaluating a latest class of radiation, propagation, and scattering issues. Applications to antenna theory and design are one of the most promising areas of fractal-electrodynamics research.

An critique of distinctfigures including Koch snowflake/islands fractal iterations, Sierpinski gasket and carpets fractal stages, fractal trees and Hilbert curves is given in [4].

This paper presents an outline of one of the standard fractal antenna called Koch fractal antenna and its implementation with different structures.

\section{KOCH FRACTAL ANTENNA}

Fractal entities have some exclusivemath properties. One of them is the possibility to bind in a finite area a very long curve. The resulting curve is Koch curve that is highly convoluted and nowhere differentiable.

The von Koch curve is a classic example of fractals. Swedish mathematician Helge von Koch [9] introduced what is termed Koch curve.Some features like bandwidth, resonance frequency, and radiation resistance of antennas have been improved by the von Koch monopole that was first reported in 1998 as the small fractal antenna. The Koch snowflake (also known as the Koch star and Koch island) is a mathematical arc, and one of the primitive fractal curves to have been described. 
The Koch curve is simply formed using are pleated method originating with the initiator of the set as the unit line segment (step $\mathrm{n}=0$ in the figure). The unit line segment is branched into thirds, and the middle third is eliminated. The middle third is then restored with two equal sections, both one-third in length, which cast an equilateral triangle $(\operatorname{step} n=1)$; this step is the generator of the curve. At the next step $(n=2)$, the middle third is detached from each of the four segments and each is recovered with two identical segments as before. This technique is imitated to infinite number of times to produce the Koch curve. Koch curve is seemingly infinite in length is one of its property. This may be seen from the construction process. [10]

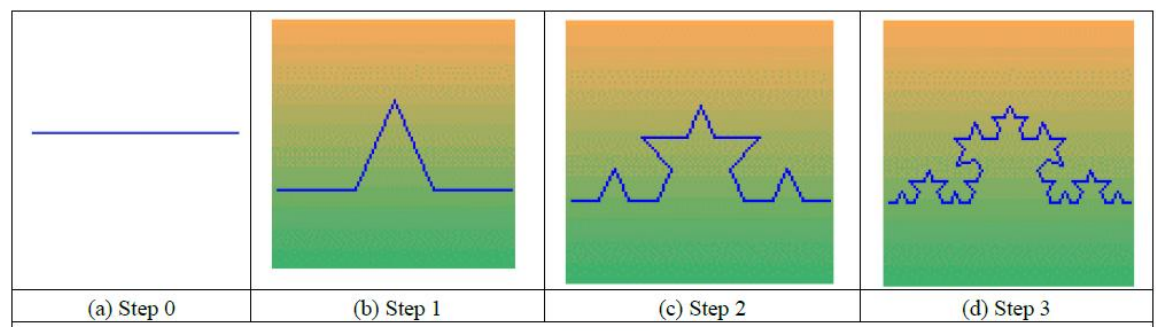

Figure 2 Iterative Construction of Koch curve

Some features of common Euclidean shapes can be improved by fractals and one such example is the Koch monopole. At each iteration its length grows as $(4 / 3)^{n}$, being a nonrectifiable curve, Koch curve has neither a piecewise continuous derivative. Its shape is highly rough and uneven which makes it nowhere differentiable. Thus, it appears as a good candidate for becoming an efficient radiator.

Self-similarity and the space-filling properties of any fractal structures are being contained and used in fractal dimension. The fractal similarity dimension (FD) is defined as

$$
F D=\frac{\log N}{\log \left(\frac{1}{\varepsilon}\right)}=\frac{\log (4)}{\log (3)}=1.26186
$$

Where $N$ is the total number of distinct copies, and $(1 / \varepsilon)$ is the reduction factor value which means how will be the length of the new side change with respect to the original side length.

Koch fractal antennas are constructed on the basis of Koch curves. A high response has been achieved by the variations on the von Koch curve, by their space-filling nature. In wireless communication the von Koch curve has proved its usefulness as antenna[11].Variants of the Koch curve have been discussed in this paper and compared.

\subsection{Isosceles Triangle}

Bow-tie antenna has been widely studied and used in engineering applications because of its ultra-broadband, light weight, thin profile configurations, low cost, easiness of fabrication, reliability and conformability.[12] One of the planar forms of UWB finite biconical antenna is a bow-tie antenna. It is afrequency self-sufficient and practical angle-dependent antenna.

Aset of isosceles triangles with Koch-like sides are found in fractal bow-tie antenna. From the initial isosceles triangle, small isosceles triangles are cut off from the center of each side. Then the methodrepeats in the tips of two sides of each angle of the rough triangle while a minor one extends from middle of equilateral sides of each isosceles-triangular notch of last iterative. The iterative procedure itself splits; forming the novel fractal bow-tie geometry, as shown in figure 3(a).The fractal bow-tie fabricated is shown in figure 3(b).
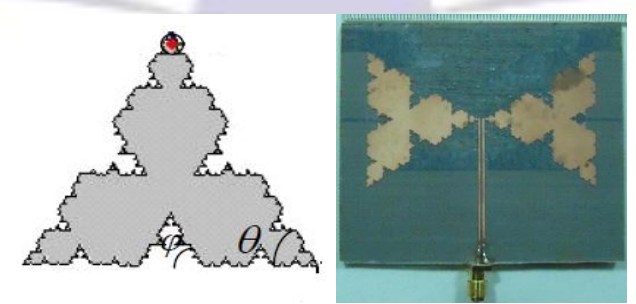

(a)(b)

Figure 3: (a) Geometry of the Koch-like sided bow-tie dipole (b) Photo of the fabricated prototype

This novel fractal bow-tie of iterative has been modeled and simulated with Ansoft HFSSv.13. It can operate in multiband as well as ultra-wideband depending on notch angle $\phi$. When notch angle extends within range of $0^{\circ}-90^{\circ}$, UWB alters into multiband regularly. Basically operating frequency band is impacted by iterative number and notch angle. Far field radiation pattern is only sensitive to base angle of the isosceles angle $\theta[13]$

Seven resonant frequencies with limited gain (3.5-7dBi) and high efficiency $(60 \%-80 \%)$ are obtained within band $0.5 \mathrm{GHz}-$ $13.5 \mathrm{GHz}$ [13]. Compared with conventional bow-tie dipole and Sierpinski gasket with the same size, this antenna has almost the same operating properties in low frequency and better radiation pattern in high frequency. So it has more 
applications and advantages and it is very attractive to UWB, GPR, PCS, WLAN, WiFi, WiMAX, and other communication systems.

\subsection{Rectangle Patch}

The patch component has been created based on a clean rectangular shape where the longitudinal mellow edges have been replaced by the fourth repetition of the Koch fractal curve. To get an increased miniaturization effect, the Koch-edge patch has been carried out in a PIFA configuration [14]. Consequently, this has reduced patch element length by $62 \%$ as compared with the simple rectangular patch. The aspired upper band (HiperLAN2) antenna nature has been accomplished by the usage of $U$-shaped slot [15]. The $U$-shape slot has been carved inside the patch material around the feeding mark (Figure. 4). For the lower frequency bands (GSM1800 and UMTS), the carve does not effect the antenna nature because the dimensions of the slot are much lesser than the wavelength. In this chassis, the active area covers the whole patch shape. For the HiperLAN2 band the influentially excited region is limited to the gut of the U-shaped slot. In the upper frequency band, the antenna behaves as a normal rectangular patch. The inner rectangular patch fits inside the fractal component so there is no need of miniaturization. The antenna without $U$-slot has been investigated in [16]. The Ensemble A software tool has been used in the creation procedure. The linear dimensions of the fractal patch have been decreased by $23.8 \%$ as compared to normalrectangular patch. Conventionally, there is decrease in the (impedance) bandwidth. Very small mobile terminals antennas can be designed using this fractal miniaturization technique. Miniaturization effects obtained with fractal patch antennas can be mixed with other techniques. To further reduce the size, shorting pins can be integrated as in PIFA architecture roughly halving its size.

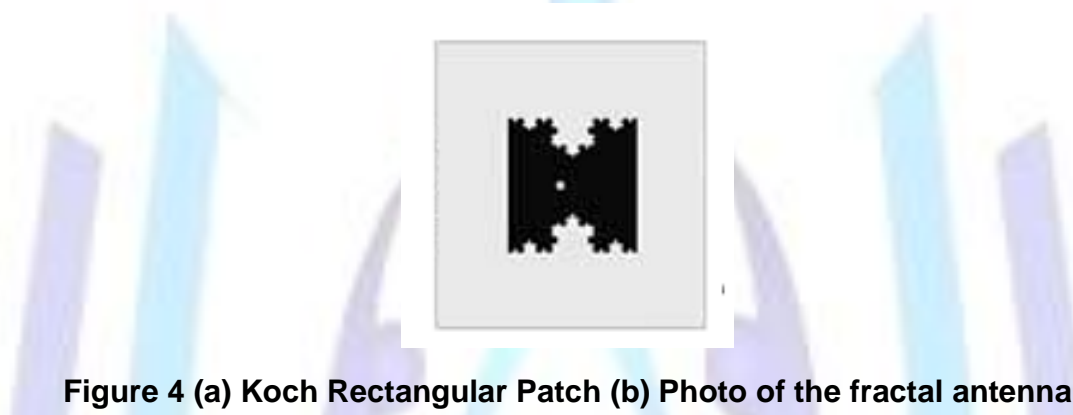

Figure 4 (a) Koch Rectangular Patch (b) Photo of the fractal antenna

The usage of multiple-input-multiple-output (MIMO) antenna techniques in mobile terminal has become one of the essential points of research committing to the objective of augmenting the scope of wireless systems. Multiple element antenna systems are thus an affair of research and these may cover diversity, smart antennas, and MIMO or spacedivision multiplex antennasystems [17]. As shown in Fig. 5, the two exact fractal PIFA elements have been given to provide physical symmetry of the antenna network.

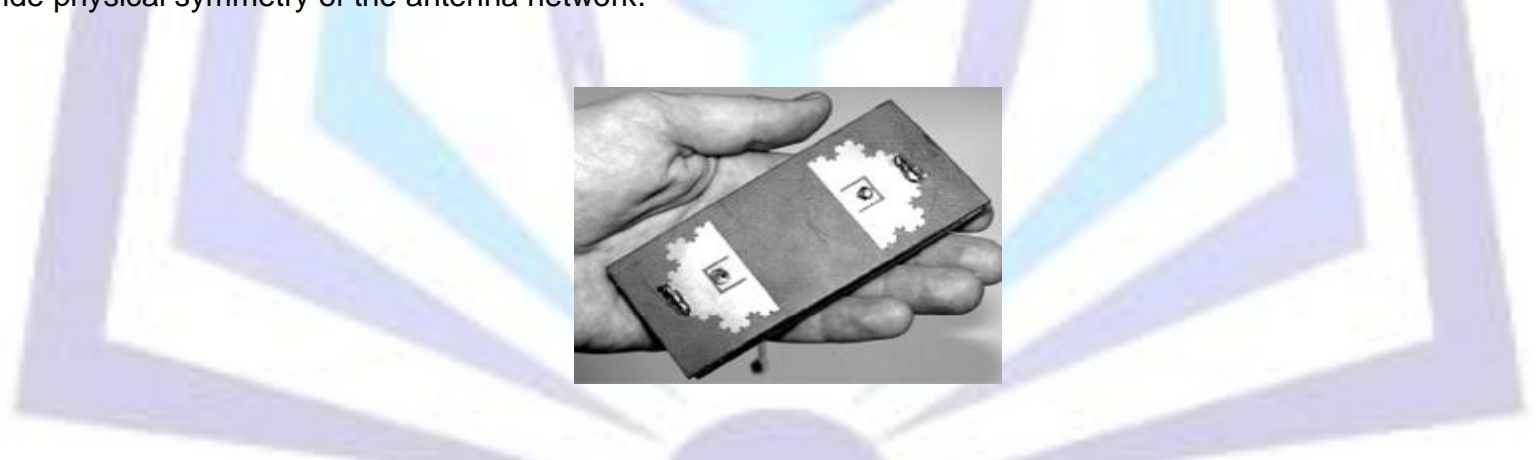

Figure 5 Photo of the two-element fracatal MIMO antenna prototype

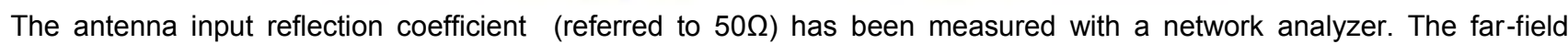
radiation patterns have been measured in an anechoic chamber.

Furthermore, it can be successfully used in compact multi-element antenna arrangements due to the very small size of the patch element. Antenna meets the requirement for mobile terminals.

\subsection{Pentagonal fractal geometry}

The plan begins with a pentagon whose sides are one unit long. In the first monotony, a triangle with sides' one-third unit long is combined in the interior of each side of the pentagon. In the second iteration, a triangle with sides' one-ninth unit long is added in the center of each side. Successive iterations extend this process considerably [18]. The first four stages are shown below in figure 7 . 


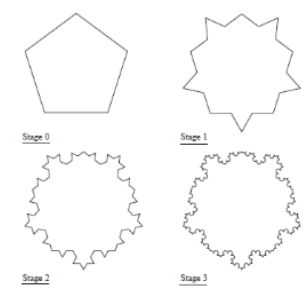

Figure 7 Stages of pentagon fractal shape

A diminished size three band Koch Pentagonal fractal antenna is presented here. The antenna benefits pentagonal shape for the basic fractalization combined with inner sides etched with Koch fractal pattern of the first iteration providing contraction in the overall size of the antenna. Koch pentagon of the first iteration is fractalized into 25 Koch pentagons as shown in figure 8. For higher order of iterations, more size reduction is accomplished, producing equal number of radiation bands. Optimization is done for achieving radiations in the S, C and X bands. Ansoft HFSS, CST Microwave Studio and Solid Works are used for the 3D Modeling. $S_{11}$ frequency optimization and radiation pattern calculations are done. The proposed third iteration fractal configuration is fabricated on Rogers RT5870 with dielectric constant 2.33[19].

With the increase in the size of first order Koch fractal, the metallic part of the antenna reduces, and the resonant frequency of the pentagon also tends to shift towards the lower frequencies. Simulations are done to analyze the exact effects of etching Koch fractal into the center of pentagon patch.

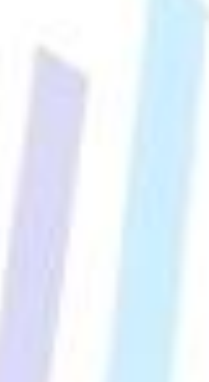

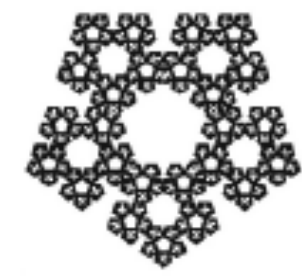

(a)

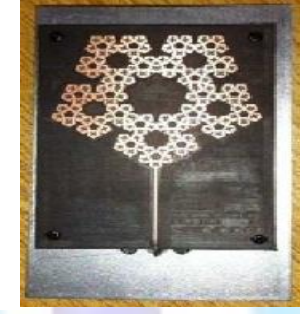

(b)

Figure 8 (a) Koch embedded pentagonal fractal geometry, (b) Fabricated prototype

Improved matching is an additional advantage of embedding the Koch fractal in the pentagon upto a certain increase in Koch fractal area and optimal value is selected for the further fractalization of the proposed third order geometry. The proposed fractal geometry is designed for resonating at the first harmonic frequency of $2.4 \mathrm{GHz}$ for the WIMAX and WIFI applications.

\section{COMPARISION OF DIFFERENT STRUCTURES}

Table 1 shows some of the parameters of comparison between the above discussed Koch fractal antennas.

Table 1 Comparison of different shapes of Koch fractal antennas

\begin{tabular}{|c|c|c|c|c|c|c|}
\hline Shape & Frequency & $\begin{array}{c}\text { Impedance } \\
\text { Match }\end{array}$ & $\begin{array}{c}\text { Dielectric } \\
\text { Constant }\end{array}$ & $\begin{array}{c}\text { Loss } \\
\text { Tangent }\end{array}$ & $\begin{array}{c}\text { Dielectric } \\
\text { Substrate }\end{array}$ & Tool \\
\hline $\begin{array}{c}\text { Isosceles } \\
\text { Triangle }\end{array}$ & $\begin{array}{c}\text { PCS, WLAN, } \\
\text { WiFi, WiFax }\end{array}$ & $50 \Omega$ & 2.2. & 0.0009 & Taconic & Ansoft HFSS \\
\hline Rectangle & $\begin{array}{c}\text { GSM, } \\
\text { UMTS, }\end{array}$ & $50 \Omega$ & 2.2 & 0.0025 & Duroid 5880 & $\begin{array}{c}\text { Ensemble } \\
\text { Ansoft }\end{array}$ \\
\hline Pentagon & WiMax, WiFi & $50 \Omega$ & 2.33 & 0.0012 & $\begin{array}{c}\text { Rogers RT } \\
5870\end{array}$ & $\begin{array}{c}\text { Ansoft } \\
\text { HFSS, CST } \\
\text { Microwave } \\
\text { Studio }\end{array}$ \\
\hline
\end{tabular}

Graph below shows a comparison between different fractals in terms of $\mathrm{S}_{11}$ (reflection coefficient). $\mathrm{S}$ parameters known as scattering parameters, they provide us with the signal reflected from the antenna. This is because gain we get is usually more important than how much the signal has been phase shifted. 


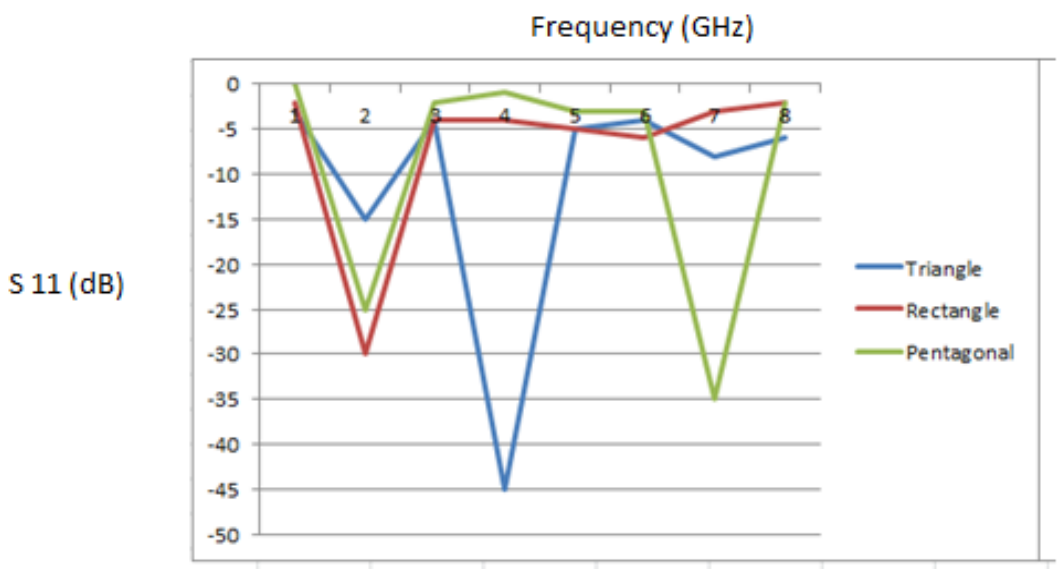

Fig 1 Comparison between $S_{11}$ of different fractal antennas

\section{CONCLUSION}

For wireless communication system, antenna is one of the most critical components. Microstrip patch antennas are widely implemented in many applications due to their attractive features such as low-profile, light weight, conformal shaping, low cost, high efficiency, and simplicity of manufacture. However the major disadvantage of the microstrip patch antenna is its inherently narrow impedance bandwidth.

Further the tremendous increase in wireless communication in the last few decades has led to the need of larger bandwidth and low profile antennas for both commercial and military applications. One technique to construct a multiband antenna is by applying fractal shape into antenna geometry.

Koch curve is a classical fractal model. The compact size of the antenna geometry makes it useful for wireless communication applications. The paper presented a review of variants of Koch curves, and their comparison.

\section{REFERENCES}

[1] Dastranj A., Imani A., and Moghaddasi M., "Printed Wide-Slot Antenna for Wideband Applications", IEEE Transactions on Antennas and Propagation, 56(10), 3097-3102, (2008).

[2] ZehforooMsh Y., Ghobadi C., and Nourinia J., "Antenna Design for Ultra Wideband Application Using a New Multilayer Structure", PIERS, 2(6), 544-549, (2006).

[3] Garg R., Bartia P., Bahl I., Ittipiboon A., "Microstrip Antenna Design Handbook", Artech House Inc. Norwood, MA, 1-68, 253-316. (2001)

[4] Werner D.H. and Ganguly S., "An Overview of Fractal Antenna Engineering Research", IEEE Antennas and Propagation Magazine, 45(1), 38-57, (2003).

[5] Puente, C., J. Romeu, R. Pous, X. Garcia, and F. Benitez, "Fractal multiband antenna based on Sierpinski gasket," Electron.Letter32(1), 1-2, (1996).

[6] Puente-Baliarda, C., J. Romeu, R. Pous, and A. Cardama, "On the behavior of the Sierpinski multiband fractal antenna," IEEETransactions on Antennas and Propagation, 46(4), 517-524, (1998).

[7] Werner D.H., Haupt R.L., and Werner P.L., "Fractal Antenna Engineering: The Theory and Design of Fractal Antenna Arrays", IEEE Antennas and Propagation Magazine, 41(5), 37-59, (1999).

[8] Mandelbrot B.B., "Fractal Geometry: What is it, and What does it do?", Proc. R. Soc. London A. 423, 3-16, (1989).

[9] Fractals are Smart: Science, Maths\& Art! , www.FractalFoundation.org.

[10] Jibrael F.J., "Multiband Cross Dipole Antenna Based on the Triangular and Quadratic Fractal Koch Curve", International Journal of Engineering, 4(3), 201-209, (2010).

[11] Sable N., Gharat S., Bhosale J., Khobragade S, "Study of Koch Monopole Fractal Antenna", General Assembly and Scientific Symposium, Istanbul, 1, (2011).

[12] Twak Y., Kabalan K.Y., Hajj A.E., Christodoulou C.G., and Coastantine J., " A Simple Multiband Printed Bowtie Antenna", IEEE Antennas and Wireless Propagation Letters, 7, 557-560, (2008).

[13] Li Daotie, Mao J-F, “ A Koch-like Sided Fractal Bow-tie Dipole Antenna”, IEEE Transactions on Antennas and Propagation (2011). 
[14] T.Taga, "Analysis of planar inverted-F antennas and antenna design for portable radio equipment", in Analysis, Design and Measurement of Small and Low-Profile Antennas, K.Hirasava and M. Heneishi, Eds. Norwood, MA: Artech House, 1992.

[15] P. Salonen, M. Keskilammi, and M. Kivikoski, "Single-feed dual-band planar inverted-F antenna with U-shaped slot," IEEE Transactions on AntennasPropagation., 48, 1262-1264, (2000).

[16] J. Guterman, A. A. Moreira, and C. Peixeiro, "Dual-band miniaturized microstrip fractal antenna for a small GSM1800 + UMTS mobile handset," in Proc. 12th IEEE Mediterranean Electro technical Conf., Dubrovnik, 499-501, (2004).

[17] S.R. Saunders, Antennas and Propagation for Wireless Communication Systems, New York: Wiley, 1999.

[18] Kumar R., Chaubey P.N., " On the Design of CPW-feed Pentagonal-Cut UWB Fractal Antenna”, International Journal of Microwave and Optical Technology, 6(5), 249-254, (2011).

[19] Khna O.M., Islam Z.U., Rashid I., Bhatti F.A., and Islam Q.U., " Novel Miniaturization Koch Pentagonal Fractal antenna for Multiband Wireless Applications”, Progress in Electromagnetics Research, 141, 693-710, (2013). 